\title{
Atomic carbon in PSS 2322+1944, a quasar at redshift 4.12^
}

\author{
J. Pety ${ }^{1,2}$, A. Beelen ${ }^{3}$, P. Cox ${ }^{3}$, D. Downes ${ }^{1}$, A. Omont ${ }^{4}$, F. Bertoldi ${ }^{5}$, and C. L. Carilli ${ }^{6}$ \\ 1 IRAM, 300 rue de la Piscine, 38406 St-Martin-d'Hères, France \\ e-mail: pety@iram.fr \\ 2 LERMA, Observatoire de Paris, 75014 Paris, France \\ ${ }^{3}$ Institut d'Astrophysique Spatiale, Université de Paris Sud, 91405 Orsay Cedex, France \\ 4 Institut d'Astrophysique de Paris, CNRS and Université de Paris VI, 98bis Bd. Arago, 75014 Paris, France \\ 5 Max-Planck-Institut für Radioastronomie, Auf dem Hügel 69, 53121 Bonn, Germany \\ ${ }^{6}$ National Radio Astronomy Observatory, PO Box, Socorro, NM 87801, USA
}

Received 22 September 2004 / Accepted 30 October 2004

\begin{abstract}
We report the detection of the ${ }^{3} \mathrm{P}_{1} \rightarrow{ }^{3} \mathrm{P}_{0}$ fine-structure line of neutral carbon in the $z=4.12$ quasar PSS 2322+1944, obtained at the IRAM Plateau de Bure interferometer. The $[\mathrm{CI}]{ }^{3} \mathrm{P}_{1}-{ }^{3} \mathrm{P}_{0}$ line is detected with a signal-to-noise ratio of $\sim 6$ with a peak intensity of $\approx 2.5 \mathrm{mJy}$ and a velocity-integrated line flux of $0.81 \pm 0.12 \mathrm{Jy} \mathrm{km} \mathrm{s}^{-1}$. Assuming an excitation temperature of $43 \mathrm{~K}$ (equal to the dust temperature), we derive a mass of neutral carbon (corrected for magnification) of $M_{\mathrm{CI}} \approx 1.2 \times 10^{7} M_{\odot}$. In PSS $2322+1944$, the cooling due to $\mathrm{C}$ is about 6 times smaller than for $\mathrm{CO}$, whereas the $\mathrm{CO}$ and $\mathrm{C}$ cooling represents $\approx 10^{-4}$ of the far-infrared continuum and more than half of the cooling due to $\mathrm{C}^{+}$.
\end{abstract}

Key words. galaxies: formation - galaxies: starburst - galaxies: high-redshift - quasars: emission lines quasars: individual: PSS 2322+1944 - cosmology: observations

\section{Introduction}

With the detection of dust and molecular gas in sources at high redshift, it has become possible to probe the physical conditions of the interstellar medium in galaxies and in the hosts of quasi-stellar objects (QSOs) at cosmological distances. The high- $z$ sources detected in ${ }^{12} \mathrm{CO}$ (to date 30 sources between $1.44<z<6.42$ - see, e.g., Greve et al. 2004) have massive reservoirs of warm and dense molecular gas (a few $10^{10}-10^{11} M_{\odot}$ ), which are predominantly excited by extreme starbursts with implied star formation rates $\approx 10^{3} M_{\odot} \mathrm{yr}^{-1}$. Multiline $\mathrm{CO}$ studies are available in only a few cases, and the detection of species other than carbon monoxide is reported in only a couple of sources. A remarkable example is the Cloverleaf, a strong gravitationally lensed QSO at $z=2.56$ where four $\mathrm{CO}$ transitions were detected, together with the two fine-structure lines of neutral carbon [CI] (Barvainis et al. 1997; Weiß et al. 2003) and the $J=1 \rightarrow 0$ transition of $\mathrm{HCN}$ (Solomon et al. 2003).

Atomic carbon is an important probe of the neutral dense gas. It is a good tracer of molecular gas in external galaxies and plays a central role in the cooling of the gas (Gérin \& Phillips $1998,2000)$. In high- $z$ sources, the detection of the $[\mathrm{CI}]$ lines enables to obtain further constraints on the physical conditions of the interstellar gas in addition to those obtained from the

^ Based on observations obtained with the IRAM Plateau de Bure interferometer.
CO transitions (Weiß et al. 2003), providing useful information on the gas column density, the thermal balance, and the UV illumination.

PSS $2322+1944$ is an optically luminous, gravitationally lensed QSO at $z=4.12$ which was studied in detail both in the dust and radio continuum emission (Omont et al. 2001; Beelen et al. 2004) and in the $J=5 \rightarrow 4,4 \rightarrow 3,2 \rightarrow 1$, and $1 \rightarrow 0$ transitions lines of CO (Cox et al. 2002; Carilli et al. 2002). With an apparent far-infrared (FIR) luminosity of $3 \times 10^{13} L_{\odot}$, it harbors a massive reservoir of molecular gas which is the site of active star formation with an implied star formation rate of $\sim 900 M_{\odot} \mathrm{yr}^{-1}$. The CO line emission is resolved into an Einstein Ring with a diameter of 1.'5, a direct indication of lensing. The amplification factor is estimated to be about 3.5. The data are consistent with a disk surrounding the QSO with a radius of $2 \mathrm{kpc}$ and a dynamical mass of a few $10^{10} M_{\odot}$ (Carilli et al. 2003).

The observations of the molecular gas in PSS 2322+1944 provide one of the best examples to date of active star formation in the host galaxy of a luminous, high redshift QSO. The strong CO emission in PSS 2322+1944 makes it a prime target to search for the ${ }^{3} \mathrm{P}_{1} \rightarrow{ }^{3} \mathrm{P}_{0}$ transition of [CI] $\left(v_{\text {rest }}=\right.$ $492.161 \mathrm{GHz}$ ) which, at $z=4.12$, is shifted into the $3 \mathrm{~mm}$ atmospheric window.

Here we report the ${ }^{3} \mathrm{P}_{1} \rightarrow{ }^{3} \mathrm{P}_{0}[\mathrm{CI}]$ line in PSS $2322+1944$. After the detection of $[\mathrm{CI}]$ in the Cloverleaf, and, recently, in IRAS FSC $10214(z=2.3)$ and SMM J14011+0252 $(z=2.5)$ 


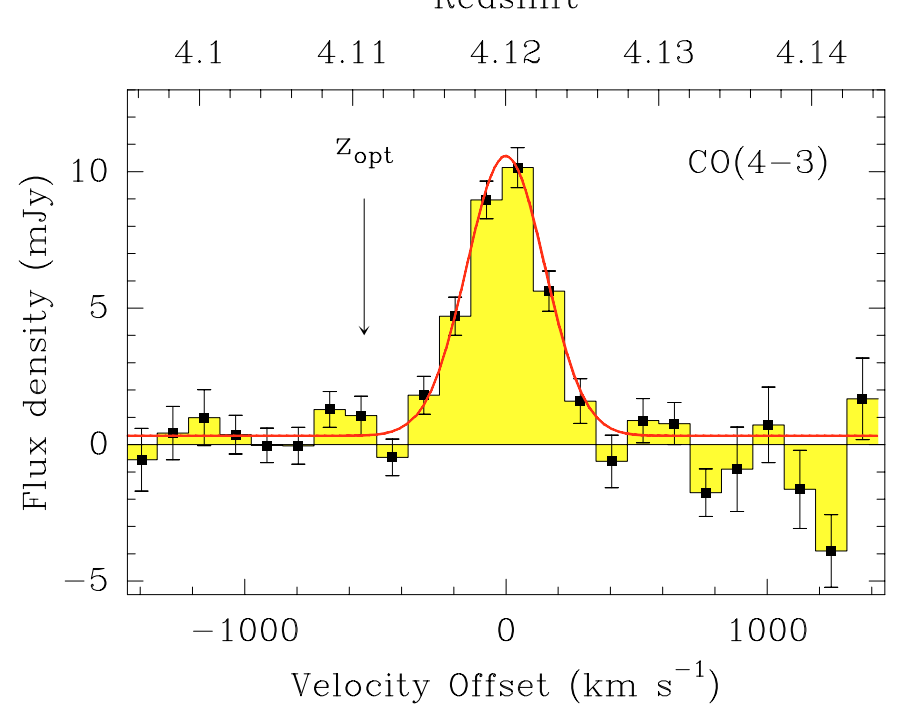

Redshift

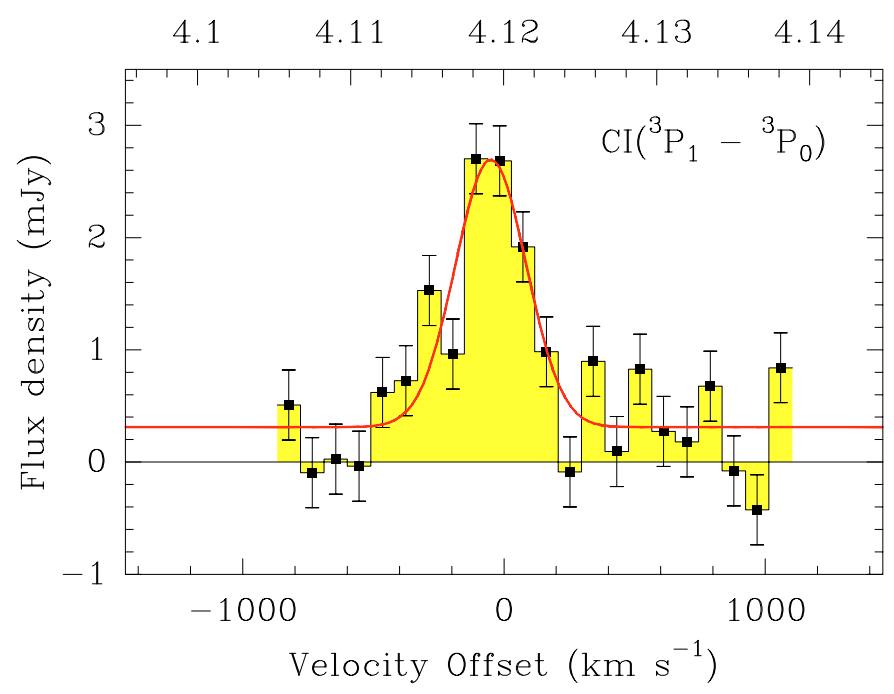

Fig. 1. Observed spectra of the $[\mathrm{CI}]]^{3} \mathrm{P}_{1} \rightarrow{ }^{3} \mathrm{P}_{0}$ (this paper) and $\mathrm{CO}(J=4 \rightarrow 3)$ lines (from Cox et al. 2002) toward the $z=4.12$ quasar PSS $2322+1944$. The black horizontal lines show the zero flux density level. The red lines show the results of Gaussian + continuum fits.

reported by Weiß et al. (2004), these observations represent the fourth clear detection of $[\mathrm{CI}]$ in a high- $z$ source. In this paper, we assume the concordance $\Lambda$-cosmology with $H_{0}=$ $71 \mathrm{~km} \mathrm{~s}^{-1} \mathrm{Mpc}^{-1}, \Omega_{\Lambda}=0.73$ and $\Omega_{m}=0.27$ (Spergel et al. 2003).

\section{Observations and data reduction}

Observations of the ${ }^{3} \mathrm{P}_{1} \rightarrow{ }^{3} \mathrm{P}_{0}$ transition of $[\mathrm{CI}]$ in PSS 2322+1944 were carried out with the IRAM Plateau de Bure interferometer (PdBI) in a series of observing sessions between July 2002 and June 2004. The total integration time of the useful data is equivalent to $34 \mathrm{~h}$ with 6 antennas. In practice, all the observing time was allocated during the summer, when antenna maintenance takes place. The total on-sky integration



Fig. 2. Map of the $[\mathrm{CI}]{ }^{3} \mathrm{P}_{1} \rightarrow{ }^{3} \mathrm{P}_{0}$ velocity-integrated emission toward PSS $2322+1944$. The emission is integrated between -400 and $+400 \mathrm{~km} \mathrm{~s}^{-1}$. The white cross indicates the optical position at RA = 23:22:07.25, Dec $=19: 44: 22.08$ (J2000.0). The contours correspond to multiples of $3 \sigma=0.34 \mathrm{mJy} / \mathrm{beam}$. Negative levels are shown as dotted contours with the same step. The synthesized beam of $6{ }^{\prime \prime} 8 \times 5^{\prime \prime} .1$ $\left(73^{\circ}\right)$ is shown in the lower left corner.

time amounts to $116 \mathrm{~h}$ often with 4 or 5 (and sometimes with 6) antennas.

We used the interferometer in the $\mathrm{D}$ configuration. The combined $3.2 \mathrm{~mm}$ data result in a synthesized beam of $6^{\prime \prime} 85 \times$ $5^{\prime \prime} 09$ at a position angle of $73^{\circ}$. The $3 \mathrm{~mm}$ receivers were tuned first at the red-shifted frequency of the $[\mathrm{CI}]{ }^{3} \mathrm{P}_{1} \rightarrow{ }^{3} \mathrm{P}_{0}$ line, i.e. $96.127 \mathrm{GHz}$ at $z=4.119$ and then at $96.063 \mathrm{GHz}$ for the second half of the data. This is the reason why the spectrum is noisier at the very ends of the band (Fig. 1). At those frequencies, the typical SSB system temperatures were $\approx 150-250 \mathrm{~K}$. The water vapor ranged between 4 and $10 \mathrm{~mm}$ on different sessions. The $580 \mathrm{MHz}$ instantaneous IF-bandwidth were observed with a resolution of $1.5 \mathrm{MHz}$.

All data reduction were done using the GILDAS softwares. Standard calibration methods using close calibrators were applied. The bandpass calibration was done on the quasar 3C 454.3. The amplitude and phase calibration were performed on $3 \mathrm{C} 454.3$ and the nearby quasar $2230+114$. Only data with phase noise better than $40 \mathrm{deg}$ were used. The maximum position errors at $3.2 \mathrm{~mm}$ introduced by such phase noise is $<0$. . 5 . The flux calibration is based on the PdBI primary calibrator MWC 349. The fluxes of 3C 454.3 (resp. 2230+114) varied from 7 to $4 \mathrm{Jy}$ (resp. 4 to $2.4 \mathrm{Jy}$ ) during the 3 years observing period.

A standard calibrated $u v$ table was produced and analyzed both by direct fits in the $u v$ plane (as this avoids the deconvolution step) and by making the deconvolved image shown in Fig. 2. Both methods confirm that the $[\mathrm{CI}]{ }^{3} \mathrm{P}_{1} \rightarrow{ }^{3} \mathrm{P}_{0}$ emission is i) detected; ii) not resolved by our observations; and iii) centered on the optical position which is also the phase center 
Table 1. Properties of the $[\mathrm{CI}]$ and $\mathrm{CO}(4 \rightarrow 3)$ lines observed toward PSS $2322+1944$.

\begin{tabular}{ccccccccc}
\hline \hline Line & $\begin{array}{c}v_{\text {rest }} \\
{[\mathrm{GHz}]}\end{array}$ & $\begin{array}{c}v_{\text {obs }} \\
{[\mathrm{mJy}]}\end{array}$ & $\begin{array}{c}\text { Peak Int. } \\
{\left[\mathrm{km} \mathrm{s}^{-1}\right]}\end{array}$ & $\begin{array}{c}\text { Position } \\
{\left[v_{\mathrm{FWHM}}\right.}\end{array}$ & $\begin{array}{c}I \\
{\left[\mathrm{Jy} \mathrm{km} \mathrm{s}^{-1}\right]}\end{array}$ & $\begin{array}{c}L^{\prime} \\
{\left[10^{10} \mathrm{~K} \mathrm{~km} \mathrm{~s}^{-1} \mathrm{pc}^{2}\right]}\end{array}$ & $\begin{array}{c}L \\
{\left[10^{8} L_{\odot}\right]}\end{array}$ \\
\hline$[\mathrm{CI}]{ }^{3} \mathrm{P}_{1} \rightarrow{ }^{3} \mathrm{P}_{0}$ & 492.161 & 96.127 & 2.4 & $-52 \pm 25$ & $319 \pm 66$ & $0.81 \pm 0.12$ & $3.4 \pm 0.5$ & $1.1 \pm 0.2$ \\
$\mathrm{CO}(4 \rightarrow 3)^{\dagger}$ & 461.041 & 90.048 & 10.3 & $-3 \pm 10$ & $348 \pm 40$ & $3.83 \pm 0.40$ & $16.3 \pm 1.5$ & $5.1 \pm 0.5$ \\
\hline
\end{tabular}

NOTE. - The $\mathrm{CO}(4 \rightarrow 3)$ data are from Cox et al. (2002). The continuum and the line were fitted separately using an a priori line window (between -400 to $+400 \mathrm{~km} \mathrm{~s}^{-1}$ ) defined from the high signal-to-noise $\mathrm{CO} J=4 \rightarrow 3$ spectrum. The luminosities are not corrected for the lens amplification $(m=3.5)$.

of the observations. The two latter points allow us to derive the source spectrum from the real part of the average of all the complex visibilities. The main advantage of this method is to directly obtain the spectrum from simple operations instead of applying the usual fitting procedures in the $u v$ plane. Any further processing (smoothing, fitting) was performed using the CLASS software.

The final [CI] spectrum at a velocity resolution of $90 \mathrm{~km} \mathrm{~s}^{-1}$ is displayed in Fig. 1 together with the spectrum of the $J=4 \rightarrow 3$ transition of CO. The image of the $[\mathrm{CI}]$ emission is shown in Fig. 2. The $[\mathrm{CI}]{ }^{3} \mathrm{P}_{1} \rightarrow{ }^{3} \mathrm{P}_{0}$ fine-structure line is clearly detected at the same redshift as the $\mathrm{CO}$ emission with a peak flux density of $S_{v}=2.69 \pm 0.31 \mathrm{mJy}$ (including the continuum flux of $0.31 \mathrm{mJy}$ ). Table 1 summarizes the line parameters derived from continuum + Gaussian fits.

\section{Results and discussion}

The integrated flux of the $[\mathrm{CI}]{ }^{3} \mathrm{P}_{1} \rightarrow{ }^{3} \mathrm{P}_{0}$ line is $0.81 \pm$ $0.12 \mathrm{Jy} \mathrm{km} \mathrm{s}^{-1}$, a factor of 5 lower than the $\mathrm{CO}(4 \rightarrow 3)$ line. A Gaussian fit yields a line width of $319 \pm 66 \mathrm{~km} \mathrm{~s}^{-1}$ and a line center displaced by $-50 \mathrm{~km} \mathrm{~s}^{-1}$ relative to $\mathrm{CO}(4 \rightarrow 3)$. Due to the weak signal to noise ratio of the $[\mathrm{CI}]$ data, the difference in the line position with the higher quality $\mathrm{CO}(4 \rightarrow 3)$ data should not be over-interpreted. The [CI] line flux implies a line luminosity of $L_{\mathrm{CI}}^{\prime}=3.4 \pm 0.5 \times 10^{10} \mathrm{~K} \mathrm{~km} \mathrm{~s}^{-1} \mathrm{pc}^{2}$ or $1.1 \pm 0.2 \times$ $10^{8} L_{\odot}$ (see, e.g., Solomon et al. 1997, for the definition of the line luminosity). The [CI] luminosity in PSS $2322+1944$ is thus about a factor 2 lower than in the case of the Cloverleaf where $L_{\mathrm{CI}}^{\prime}=6.1 \times 10^{10} \mathrm{~K} \mathrm{~km} \mathrm{~s}^{-1} \mathrm{pc}^{2}$, as derived from the $[\mathrm{CI}]$ velocity-integrated flux of Barvainis et al. (1997).

The continuum emission is detected at $96 \mathrm{GHz}$ with $0.31 \pm$ $0.08 \mathrm{mJy}$, which is consistent for dust emission with the available photometric data at higher frequency. The FIR spectral energy distribution of PSS $2322+1944$ is well reproduced with a grey body of temperature $43 \pm 6 \mathrm{~K}$ and a dust emissivity $\propto v^{1.6 \pm 0.3}$ (Beelen et al. 2004).

The detection of the $[\mathrm{CI}]{ }^{3} \mathrm{P}_{1} \rightarrow{ }^{3} \mathrm{P}_{0}$ emission line in PSS 2322+1944 allows us to estimate the mass of neutral carbon $M_{\mathrm{CI}}$. Since the ${ }^{3} \mathrm{P}_{2} \rightarrow{ }^{3} \mathrm{P}_{1}$ transition (at $v_{\text {rest }}=$ $809.342 \mathrm{GHz}$ ) is not observed, we assume that the ${ }^{3} \mathrm{P}_{1} \rightarrow{ }^{3} \mathrm{P}_{0}$ transition is optically thin as is the case for the Cloverleaf (Weiß et al. 2003). Under these assumptions, we can express the mass of neutral carbon as a function of $L_{\mathrm{CI}}^{\prime}$

$M_{\mathrm{CI}}=5.65 \times 10^{-4} \frac{Q}{3} \mathrm{e}^{\left(23.6 / T_{\mathrm{ex}}\right)} L_{\mathrm{CI}\left({ }^{3} \mathrm{P}_{1} \rightarrow{ }^{3} \mathrm{P}_{0}\right)}^{\prime} M_{\odot}$, where $T_{\mathrm{ex}}$ is the excitation temperature and $Q$ is the CI partition function. Assuming that $T_{\mathrm{ex}}$ is equal to the temperature of the warm dust $T_{\text {dust }}=43 \mathrm{~K}$, the mass of neutral carbon in PSS $2322+1944$ amounts to $M_{\mathrm{CI}}=4.3 \times 10^{7} M_{\odot}$, or $1.2 \times 10^{7} M_{\odot}$ after correction for lens amplification $(m=3.5)$. Although the excitation temperature of the neutral carbon can be different than $T_{\text {dust }}$ (as observed for the Cloverleaf - see Weiß et al. 2003), the derived mass depends only weakly on $T_{\text {ex }}$. For a range of [CI] excitation temperatures from 30 to $100 \mathrm{~K}$, the mass of neutral carbon in PSS $2322+1944$ would vary from 1.2 to $1.4 \times 10^{7} M_{\odot}$.

Compared to the mass of molecular gas of $M_{\mathrm{H}_{2}}=7 \times$ $10^{10} M_{\odot}$ after correcting for amplification (Cox et al. 2002; Carilli et al. 2003), the derived mass of [CI] implies a carbon abundance relative to $\mathrm{H}_{2}$ of $[\mathrm{CI}] /\left[\mathrm{H}_{2}\right] \approx 3 \times 10^{-5}$, indicating near to solar abundances in this high-redshift system. Similar values are derived by Weiß et al. (2004). This relative carbon abundance is close to the maximum value of $2.2 \times 10^{-5}$ found for Galactic dense molecular clouds with opacities of 4 $11 \mathrm{mag}$, a value which does not vary within a factor of a few for larger $A_{\mathrm{V}}$ 's (Frerking et al. 1989).

The $[\mathrm{CI}]{ }^{3} \mathrm{P}_{1} \rightarrow{ }^{3} \mathrm{P}_{0}$ and ${ }^{3} \mathrm{P}_{2} \rightarrow{ }^{3} \mathrm{P}_{1}$ lines are major gas coolants. In the Cloverleaf, the ${ }^{3} \mathrm{P}_{2} \rightarrow{ }^{3} \mathrm{P}_{1}$ line is 2.2 times stronger than the ${ }^{3} \mathrm{P}_{1} \rightarrow{ }^{3} \mathrm{P}_{0}$ line (Weiß et al. 2003). To estimate the carbon cooling, we may assume a similar ratio for PSS $2322+1944$. To compare this to the CO cooling, we added the observed CO line luminosities of PSS 2322+1944 up to $J=9-8$, where for the unobserved transitions we adopt the prediction of the LVG model of Carilli et al. (2002). In PSS $2322+1944$, we find that the $\mathrm{CO} /[\mathrm{CI}]$ luminosity ratio is 6 , as compared to a ratio of 20 for the Cloverleaf (see Table 2). However, the total mass of $[\mathrm{CI}]$ remains somewhat smaller than that of CO (see Carilli et al. 2002).

Compared to the far-IR luminosity of PSS $2322+1944$, $L_{\mathrm{FIR}} \sim 8.6 \times 10^{12} L_{\odot}$ (corrected for lensing), the $\mathrm{CO}$ and [CI] cooling represents $\sim 10^{-4}$ of the far-IR continuum, again not very different from the ratio of $4 \times 10^{-4}$ derived for the Cloverleaf (Table 2). The recent search of the red-shifted [CII] fine-structure line in PSS 2322+1944 implies an upper limit to the [CII] line luminosity of $1.7 \times 10^{9} L_{\odot}$, a weakness which is typical for high- $z$ IR luminous galaxies (Benford et al. 2004). The CO and C cooling is therefore more than half of the cooling due to $\mathrm{C}^{+}$. The [CII] line remains the main cooling line of the gas. 
Table 2. Comparison of the interstellar gas and dust luminosities in Infrared Luminous Galaxies and in the Galactic Center.

\begin{tabular}{|c|c|c|c|c|c|c|c|c|c|}
\hline Source & $z$ & $\begin{array}{c}\mathrm{C}^{\dagger} \\
{\left[L_{\odot}\right]}\end{array}$ & $\begin{array}{l}\mathrm{CO}^{\dagger \dagger} \\
{\left[L_{\odot}\right]}\end{array}$ & $\begin{array}{c}\mathrm{C}^{+} \\
{\left[L_{\odot}\right]}\end{array}$ & $\begin{array}{l}L_{\mathrm{FIR}} \\
{\left[L_{\odot}\right]} \\
\end{array}$ & $\mathrm{CO} / \mathrm{C}$ & $(\mathrm{C}+\mathrm{CO}) / L_{\mathrm{FIR}}$ & $\mathrm{C}^{+} / L_{\mathrm{FIR}}$ & Ref. \\
\hline PSS $2322+1944^{(a)}$ & 4.12 & $1.0 \times 10^{8}$ & $6.8 \times 10^{8}$ & $\leq 1.7 \times 10^{9}$ & $8.6 \times 10^{12}$ & 6.6 & $9 \times 10^{-5}$ & $\leq 2 \times 10^{-4}$ & [1] \\
\hline Cloverleaf ${ }^{(a)}$ & 2.56 & $7.1 \times 10^{7}$ & $1.5 \times 10^{9}$ & - & $4.2 \times 10^{12}$ & 21.7 & $3.9 \times 10^{-4}$ & - & [2] \\
\hline Arp220 & 0.018 & $1.8 \times 10^{7}$ & $3.9 \times 10^{7}$ & $1.6 \times 10^{9}$ & $1.2 \times 10^{12}$ & 2.2 & $4.7 \times 10^{-5}$ & $1.3 \times 10^{-3}$ & [3] \\
\hline NGC 253 & 0.0008 & $1.6 \times 10^{5}$ & $1.6 \times 10^{6}$ & $7.8 \times 10^{6}$ & $1.0 \times 10^{10}$ & 10.0 & $1.8 \times 10^{-4}$ & $7.8 \times 10^{-4}$ & [4] \\
\hline Galactic center $^{(b)}$ & - & $6.7 \times 10^{4}$ & $2.6 \times 10^{5}$ & $2.6 \times 10^{6}$ & $3.9 \times 10^{8}$ & 3.8 & $8.4 \times 10^{-4}$ & $6.7 \times 10^{-3}$ & [5] \\
\hline
\end{tabular}

NOTE. $-{ }^{\dagger}$ Total C I luminosity; ${ }^{\dagger \dagger}$ CO luminosity up to $J=8$; ${ }^{(a)}$ the luminosities are corrected for lensing: $m=3.5$ for PSS $2322+1944$ (Carilli et al. 2003) and $m=11$ for the Cloverleaf (Venturini \& Solomon 2003); ${ }^{(b)}$ the central $5 \times 1$ deg $^{2}$ of the Galaxy. References: [1] This paper - [2] Weiß et al. (2003), Barvainis et al. (1997), A. Weiß (private communication) - [3] Gérin \& Phillips (1998) - [4] Bayet et al. (2004) - [5] Fixsen et al. (1999).

Finally, the $[\mathrm{CI}]$ luminosity relative to the integrated farIR luminosity is a good measure of the intensity of the non-ionizing UV radiation field in galaxies, because in photodissociation regions the column density of neutral carbon is mostly insensitive to the UV field, whereas the far-IR emission is directly proportional to the strength of the UV field (see, e.g., Kaufman et al. 1999; Gérin \& Phillips 2000). For PSS $2322+1944$, this ratio is $\sim 3 \times 10^{-6}$ indicating a UV radiation field of a few 1000 times larger than in the solar vicinity. Both this ratio and the implied strength of the UV illumination are comparable to the values derived for the other IR luminous galaxies and the galactic center listed in Table 2.

As in the case for local starburst galaxies such as NGC 253, $\mathrm{CO}$ is a more important coolant than C in both PSS $2322+1944$ and the Cloverleaf by about one order of magnitude (Table 2) see also Schilke et al. (1993) and Bayet et al. (2004). Similarly, in starburst galaxies or in galactic nuclei (including the Milky Way), the molecular gas is warm therefore populating the higher $\mathrm{CO}$ levels which contribute to the cooling.

The detection of the red-shifted $[\mathrm{CI}]{ }^{3} \mathrm{P}_{1} \rightarrow{ }^{3} \mathrm{P}_{0}$ transition line in the gravitationally lensed $z=4.12$ QSO PSS $2322+1944$ enables to further constrain the physical conditions of the neutral gas and to compare the major line $\left(\mathrm{C}, \mathrm{CO}\right.$ and $\left.\mathrm{C}^{+}\right)$and far-IR luminosities in this high- $z$ galaxy. Together with the recent $[\mathrm{CI}]$ detections in other sources at high redshift (Weiß et al. 2004), these results illustrate the potential of studying neutral carbon or species other than $\mathrm{CO}$ in high- $z$ sources, a field which will clearly fully develop as soon as more sensitive submillimeter arrays, such as the Atacama Large Millimeter Array (ALMA), will become operational.

Acknowledgements. We thank the IRAM Plateau de Bure staff for their support in the observations. IRAM is supported by INSU/CNRS (France), MPG (Germany), and IGN (Spain). Estelle Bayet and the referee, Dr. Phil M. Solomon, are kindly acknowledged for helpful comments.

\section{References}

Barvainis, R., Maloney, P., Antonucci, R., \& Alloin, D. 1997, ApJ, 484, 695

Bayet, E., Gérin, M., Phillips, T. G., \& Contursi, A. 2004, A\&A, 427, 45

Beelen, A., Benford, D., Cox, P., et al. 2004, ApJ, submitted

Benford, D., Cox, P., et al. 2004, ApJ, submitted

Bertoldi, F., Carilli, C., Cox, P., et al. 2003a, A\&A, 406, L55

Bertoldi, F., Cox, P., Neri, R., et al. 2003b, A\&A, 409, L47

Carilli, C. L., Cox, P., Bertoldi, F., et al. 2002, ApJ, 575, 145

Carilli, C. L., Lewis, G. F., Djorgovski, S. G., et al. 2003, Science, 300,773

Cox, P., Omont, A., Djorgovski, S., et al. 2002, A\&A, 387, 406

Gérin, M., \& Phillips T. G. 1998, ApJ, 509, L17

Gérin, M., \& Phillips T. G. 2000, ApJ, 537, 644

Greve, T., Bertoldi, F., Smail, I., et al. 2004, MNRAS, submitted

Fixsen, D. J., Bennett, C. L., \& Mather, J. C. 1999, ApJ, 526, 207

Frerking, M. A., Keene, J., Blake, G. A., \& Phillips, T. G. 1989, ApJ, 344,311

Kaufman, M. J., Wolfire, M. G., Hollenbach, D. J., \& Luhman, M. L. 1999, ApJ, 527, 795

Omont, A., Cox, P., Bertoldi, F. 2001, A\&A, 374, 371

Schilke, P., Carlstrom, J. E., Keene, J., \& Phillips, T. G. 1993, ApJ, 417, L67

Solomon, P. M., Downes, D., Radford, S. J., \& Barrett, J. W. 1997, ApJ, 478, 14

Solomon, P. M., Vanden Bout, P. A., Carilli, C. L., \& Guélin, M. 2003 , Nature, 426, 636

Spergel, D. N., Verde, L., Peiris, H., et al. 2003, ApJS, 148,175

Venturini, S., \& Solomon, P. M. 2003, ApJ, 590, 740

Weiß, A., Henkel, C., Downes, D., \& Walter, F. 2003, A\&A, 409, L41

Weiß, A., Downes, D., Henkel, C., \& Walter, F. 2004, A\&A, in press 